\title{
Integrative bioinformatics approaches for identifying potential biomarkers and pathways involved in non-obstructive azoospermia
}

\author{
Tengfei Hu${ }^{1}$, Shaoge Luo $^{1}$, Yu Xi ${ }^{1}$, Xuchong Tu ${ }^{1}$, Xiaojian Yang ${ }^{1}$, Hui Zhang ${ }^{1}$, Jiarong Feng ${ }^{1}$, \\ Chunlin Wang ${ }^{2}$, Yan Zhang ${ }^{1}$
}

${ }^{1}$ Department of Infertility and Sexual Medicine, The Third Affiliated Hospital, Sun Yat-sen University, Guangzhou, China; ${ }^{2}$ Department of Andrology, Ruikang Hospital Affiliated to Guangxi University of Traditional Chinese Medicine, Nanning, China

Contributions: (I) Conception and design: T Hu; (II) Administrative support: Y Zhang, X Yang; (III) Provision of study materials or patients: T Hu, Y Zhang; (IV) Collection and assembly of data: T Hu, S Luo; (V) Data analysis and interpretation: T Hu, S Luo; (VI) Manuscript writing: All authors; (VII) Final approval of manuscript: All authors.

Correspondence to: Yan Zhang. Department of Infertility and Sexual medicine, The Third Affiliated Hospital, Sun Yat-sen University, No. 600, Tianhe Road, Guangzhou 510630, China. Email: zhxml@sina.com.

Background: Non-obstructive azoospermia (NOA) is a disease related to spermatogenic disorders. Currently, the specific etiological mechanism of NOA is unclear. This study aimed to use integrated bioinformatics to screen biomarkers and pathways involved in NOA and reveal their potential molecular mechanisms.

Methods: GSE145467 and GSE108886 gene expression profiles were obtained from the Gene Expression Omnibus (GEO) database. The differentially expressed genes (DEGs) between NOA tissues and matched obstructive azoospermia (OA) tissues were identified using the GEO2R tool. Common DEGs in the two datasets were screened out by the VennDiagram package. For the functional annotation of common DEGs, DAVID v.6.8 was used to perform Gene Ontology (GO) and the Kyoto Encyclopedia of Genes and Genomes (KEGG) pathway enrichment analysis. In accordance with data collected from the Search Tool for the Retrieval of Interacting Genes/Proteins (STRING) database, a protein-protein interaction (PPI) network was constructed by Cytoscape. Cytohubba in Cytoscape was used to screen the hub genes. Furthermore, the hub genes were validated based on a separate dataset, GSE9210. Finally, potential micro RNAs (miRNAs) of hub genes were predicted by miRWalk 3.0.

Results: A total of 816 common DEGs, including 52 common upregulated and 764 common downregulated genes in two datasets, were screened out. Some of the more important of these pathways, including focal adhesion, PI3K-Akt signaling pathway, cell cycle, oocyte meiosis, AMP-activated protein kinase (AMPK) signaling pathway, FoxO signaling pathway, and Huntington disease, were involved in spermatogenesis. We further identified the top 20 hub genes from the PPI network, including CCNB2, DYNLL2, HMMR, NEK2, KIF15, DLGAP5, NUF2, TTK, PLK4, PTTG1, PBK, CEP55, CDKN3, CDC25C, MCM4, DNAI1, TYMS, PPP2R1B, DNAI2, and DYNLRB2, which were all downregulated genes. In addition, potential miRNAs of hub genes, including hsa-miR-3666, hsa-miR-130b-3p, hsa-miR-15b-5p, hsamiR-6838-5p, and hsa-miR-195-5p, were screened out.

Conclusions: Taken together, the identification of the above hub genes, miRNAs and pathways will help us better understand the mechanisms associated with NOA, and provide potential biomarkers and therapeutic targets for NOA.

Keywords: Non-obstructive azoospermia (NOA); expression profiling data; functional enrichment analysis; protein-protein interactions; biomarkers 
Submitted Jun 23, 2020. Accepted for publication Oct 30, 2020.

doi: $10.21037 /$ tau-20-1029

View this article at: http://dx.doi.org/10.21037/tau-20-1029

\section{Introduction}

Infertility is defined as the inability to conceive within 1 year of unprotected intercourse (1). Studies have shown that about $10-15 \%$ of couples have fertility problems, and male factors are responsible for $50 \%$ of infertility cases $(2,3)$. The causes of male infertility are complex. Azoospermia, which causes $10-20 \%$ of male infertility cases (4), is a type of male infertility in which sperm is absent. Types of azoospermia include obstructive azoospermia $(\mathrm{OA})$ and non-obstructive azoospermia (NOA) $(5,6)$. OA is mainly caused by obstruction of the posterior reproductive tract of the testis, while NOA is caused by the dysfunction of spermatogenesis. NOA is the most severe form of male infertility, with an incidence rate of $10 \%$ (7).

Human spermatogenesis essentially occurs in three stages: spermatogenic mitosis, spermatogenic meiosis, and spermatogenesis (8). Problems at any of these stages can cause sperm production to fail. While it is possible to obtain sperm of through testicular aspiration or testicular sperm extraction via microdissection, this is not feasible for the vast majority of NOA patients (9). Furthermore, the primary mechanism regulating spermatogenesis in NOA patients remains unclear (10).

In the present study, we downloaded the expression profile datasets, GSE145467 and GSE108886, from the Gene Expression Omnibus (GEO) database. We then screened out common differentially expressed genes (DEGs) using combined GEO2R and VennDiagram package analyses. We performed Gene Ontology (GO) annotation and Kyoto Encyclopedia of Genes and Genomes (KEGG) pathway enrichment analyses of common DEGs. The protein-protein interaction (PPI) network was constructed by Cytoscape, and the hub genes were obtained using the Cytohubba plug-in of Cytoscape. In addition, potential micro RNAs (miRNAs) of hub genes were predicted by miRWalk 3.0. It is hoped the results of this study can provide insights into the molecular mechanism of NOA and identify potential biomarkers and therapeutic targets. We present our findings in accordance with the STROBE and MDAR reporting checklists (available at http://dx.doi. org/10.21037/tau-20-1029).

\section{Methods}

\section{Microarray data source}

As all the data in this study were from the GEO public database (https://www.ncbi.nlm.nih.gov/geo/), the approval of the local ethics committee was not required.

We used the keywords "non-obstructive azoospermia" and "expression profiling by array" and "Homo sapiens" in the GEO database (https://www.ncbi.nlm.nih.gov/geo/) to search the mRNA expression dataset of NOA. Through retrieval, we downloaded the GSE145467 and GSE108886 expression profile datasets. The GSE145467 dataset, which was contributed by Hodžić et al. (11), is based on the GPL4133 platform of the Agilent-014850 Whole Human Genome Microarray 4x44K G4112F (Feature Number version) and includes $10 \mathrm{NOA}$ samples and $10 \mathrm{OA}$ testicular samples. The GSE108886 dataset, which was contributed by Baksi et al., is based on the GPL10558 platform of the Illumina HumanHT-12 V4.0 expression beadchip which contains eight NOA samples and four OA samples (including one testicular control sample).

\section{Screening for DEGs}

The GEO2R tool (https://www.ncbi.nlm.nih.gov/geo/ geo2r/) was used to screen DEGs in NOA tissues compared with matched OA tissues. The $t$-test and BenjaminiHochberg method were used to calculate the P value and false discovery rate (FDR), respectively. The DEGs were screened out according to FDR $<0.05$ and $|\mathrm{FC}| \geq 2.5$. The common DEGs in the two datasets were screened out by the VennDiagram package.

\section{GO and KEGG enrichment analysis}

GO functional and KEGG pathway enrichment analysis was conducted to determine the functions of common DEGs using the Database for Annotation Visualization and Integrated Discovery (DAVID; https://david.ncifcrf.gov/). The results of GO and KEGG pathway enrichment analyses were downloaded as a TXT file for subsequent analysis. The results were visualized using $\mathrm{R}$ software version 3.6.2. 
A P value $<0.05$ was set to represent a statistically significant difference.

\section{PPI network and hub gene identification}

The Search Tool for the Retrieval of Interacting Genes database (STRING; https://string-db.org/cgi/input.pl) is an online tool for analyzing the PPI information. We constructed a PPI network of common DEGs using the STRING based on a minimum required interaction score of 0.7. We then used Cytoscape software v3.7.1 (https:// cytoscape.org/) to visualize the PPI network derived from the STRING database. Using the cytoHubba plugin in Cytoscape, the nodes in the PPI network were ranked according to the degree calculation method (12) with the top 20 genes being considered the hub genes. GO and KEGG pathway analyses for the hub genes were performed using the WebGestalt (http://www.webgestalt.org/). A $\mathrm{P}$ value $<0.05$ was considered a statistically significant difference.

\section{Analysis of bub genes in the NOA subgroup}

The NOA samples in the GSE108886 dataset were analyzed according to its two subgroups: the non-obstructive azoospermia with meiotic arrest (NOA-MA) subgroup contains five samples, and the non-obstructive azoospermia with pre-meiotic arrest (NOA-PreMA) subgroup contains three samples. The Wilcox test was used to determine whether there were differences in the expression of hub genes between the two subgroups. A $\mathrm{P}$ value $<0.05$ was considered statistically significant.

\section{Validation of the hub genes}

To further verify the differential expression of hub genes, we downloaded the GSE9210 dataset, which contains 47 NOA samples and $11 \mathrm{OA}$ samples. The expression levels of genes in this dataset have been processed by lowessnormalized natural log [Cy5/Cy3] (13). The Wilcox test was used to compare the differential expression of hub genes between the NOA and OA samples in the GSE9210 dataset. A $\mathrm{P}$ value $<0.05$ was considered a statistically significant difference.

\section{Screening to regulate bub genes}

Twenty hub genes associated with NOA were imported into the miRWalk 3.0 software (http://mirwalk.umm.uniheidelberg.de/) to screen for the miRNAs that regulate target genes. The miRWalk 3.0 software integrated the prediction results of TargetScan, and a score $>0.8$ was used as the cutoff criterion. Following this, a miRNAgene regulatory network was constructed and visualized by Cytoscape. Moreover, miRNAs which targeted more than two genes were selected.

\section{Statistical analysis}

We performed R software version 3.6.2 for statistical analysis. The Wilcox test was used to compare the two groups. A P value $<0.05$ was considered a statistically significant difference.

\section{Results}

\section{Identification of DEGs in NOA}

For the GSE145467 dataset, 3,549 DEGs were identified, including 951 upregulated and 2,598 downregulated genes. For the GSE108886 dataset, 1,063 DEGs were identified, including 155 upregulated and 908 downregulated genes. VennDiagram analysis was performed to determine the intersection of the two datasets of DEGs. A total of 816 common DEGs were identified, including 52 common upregulated and 764 common downregulated genes (Figure 1 and Table S1).

\section{GO enrichment analysis}

GO enrichment analysis of the common DEGs included the following three parts: biological process (BP), molecular function (MF), and cell component (CC). We imported the common DEGs into the DAVID online analysis tool for GO enrichment analysis. This showed that for BP, common upregulated DEGs were significantly associated with extracellular matrix (ECM) organization, negative regulation of cell proliferation and apoptotic process, cell adhesion, and cellular protein metabolic process. Common downregulated DEGs were significantly associated with spermatogenesis, multicellular organism development, cell differentiation, spermatid development, and sperm motility. Common upregulated DEGs that were significantly associated with CC included extracellular exosome, extracellular space, extracellular region, ECM, and proteinaceous ECM. Common downregulated DEGs 


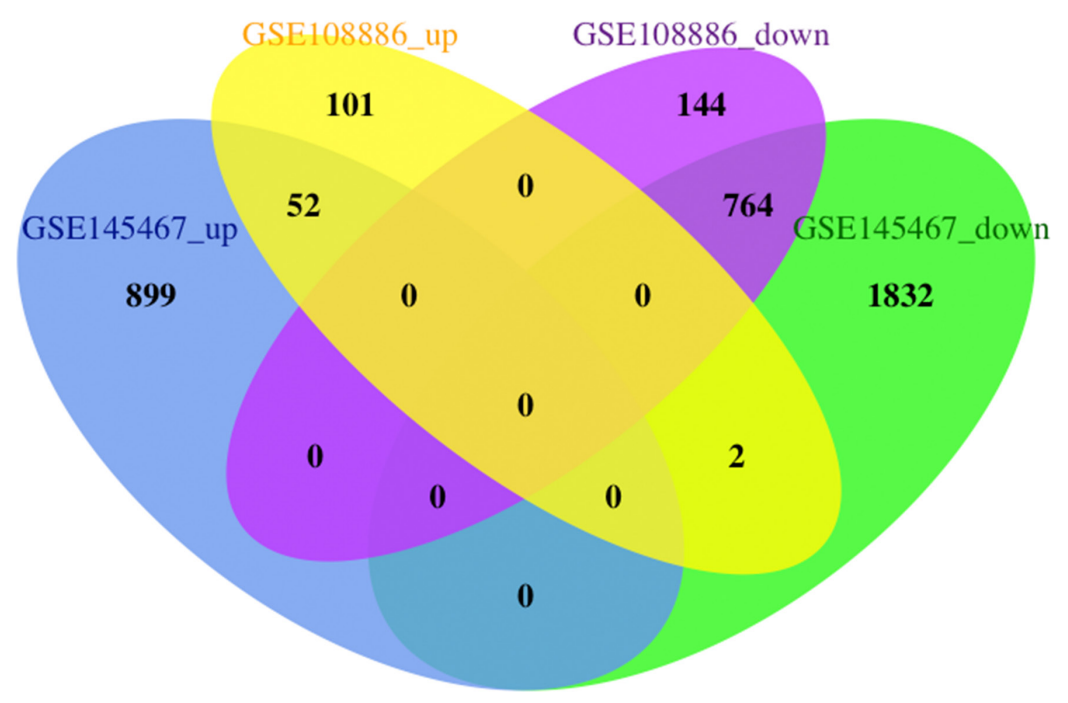

Figure 1 Identification of 816 (52 upregulated and 764 downregulated) common differentially expression genes (DEGs) from GSE145467 and GSE108886 microarray profile datasets. The FDR $<0.05$ and $\mid \mathrm{FCl} \geq 2.5$ as the cut-off criterion. FDR, false discovery rate; FC, fold change.
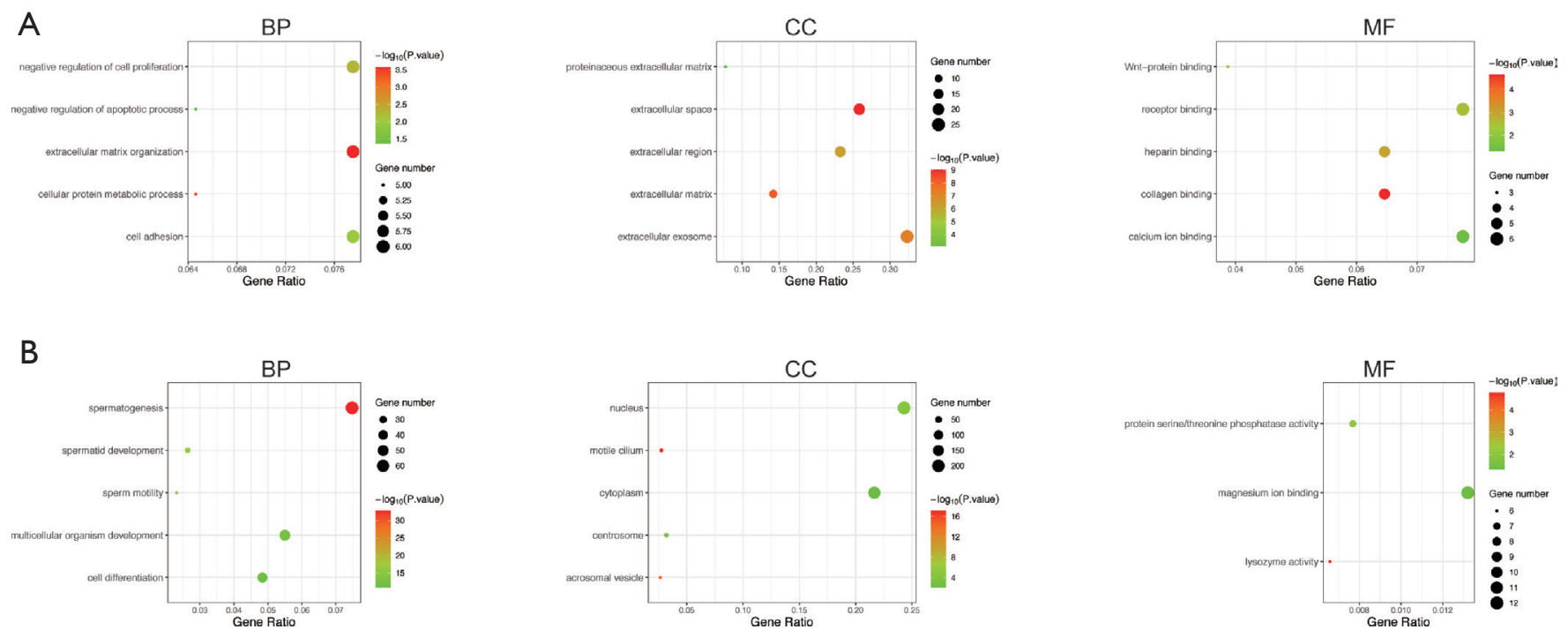

Figure 2 Gene ontology analysis of the common DEGs. (A) Common upregulated DEGs. (B) Common downregulated DEGs. BP, biological process; CC, cellular component; MF, molecular function; DEGs, differentially expressed genes.

that were significantly associated with CC included nucleus, cytoplasm, centrosome, motile cilium, and acrosomal vesicle. GO MF showed that common upregulated DEGs were significantly associated with receptor binding, calcium ion binding, collagen binding, heparin binding, and Wnt- protein binding. Common downregulated DEGs were significantly associated with protein serine/threonine kinase activity, magnesium ion binding, protein serine/threonine phosphatase activity, lysozyme activity, and microtubule motor activity. These results are shown in Figure 2 and Table 1. 
Table 1 Gene ontology analysis of common upregulated and downregulated DEGs

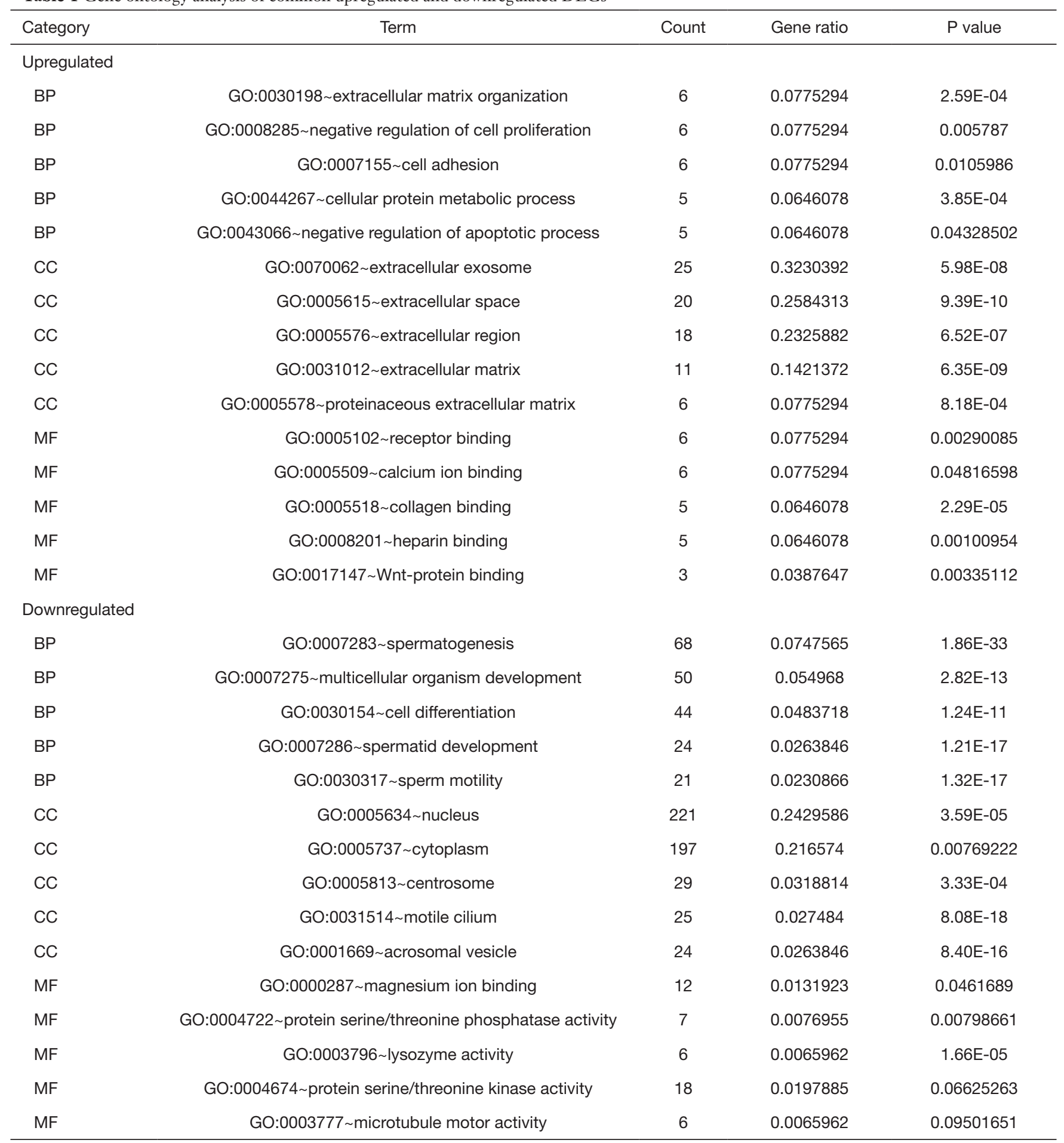

GO, gene ontology; DEGs, differentially expressed genes; BP, biological process; CC, cellular component; MF, molecular function; Count, number of DEGs. 
A

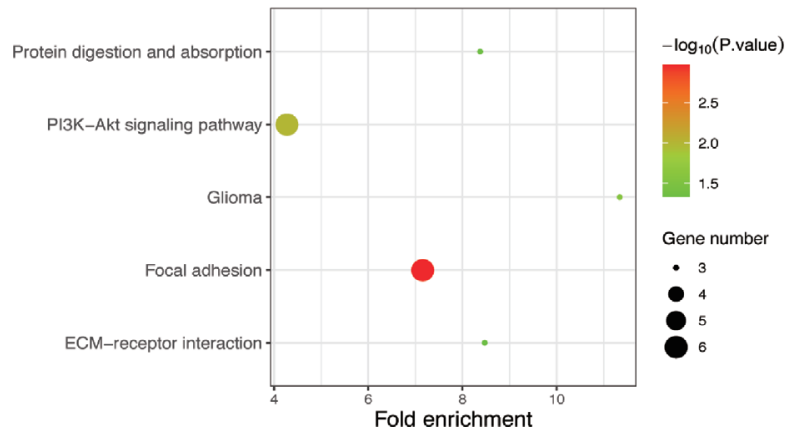

B

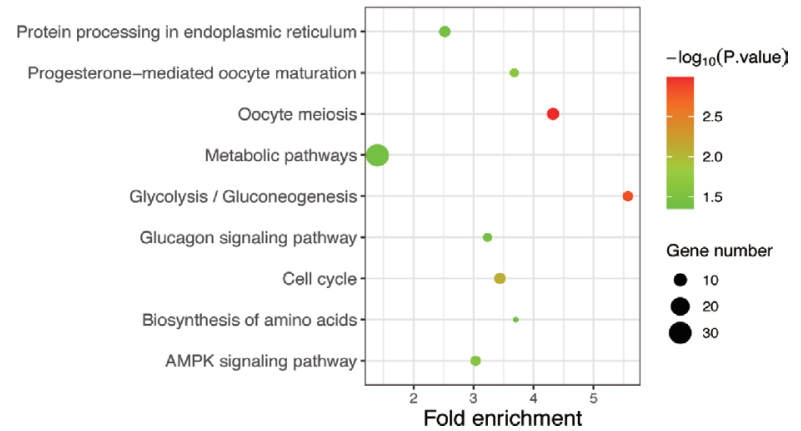

Figure 3 KEGG pathway enrichment analysis of the common DEGs. (A) Common upregulated DEGs. (B) Common downregulated DEGs. KEGG, Kyoto Encyclopedia of Genes and Genomes; DEGs, differentially expression genes.

\section{KEGG pathway enrichment analysis}

The KEGG pathway of the common upregulated and downregulated DEGs was analyzed by the DAVID database. The common upregulated DEGs were mainly involved in focal adhesion, PI3K-Akt signaling pathway, glioma, ECMreceptor interaction, and protein digestion and absorption. The common downregulated DEGs were mainly involved in oocyte meiosis, glycolysis/gluconeogenesis, cell cycle, progesterone-mediated oocyte maturation, AMP-activated protein kinase (AMPK) signaling pathway, glucagon signaling pathway, metabolic pathways, protein processing in the endoplasmic reticulum, the and biosynthesis of amino acids. These results are shown in Figure 3 and Table 2.

\section{PPI network and bub gene analysis}

According to the STRING database, the PPI network of DEGs was constructed, with 328 nodes and 604 edges being mapped as presented in Figure 4A. The top 20 hub genes were evaluated using the Degree algorithm of the Cytohubba plug-in, as shown in Figure $4 B$ and Table 3. GO and KEGG enrichment analyses of the 20 hub genes were performed using WebGestalt. As shown in Figure 5, the GO enrichment analysis was mainly involved in metabolic process, cellular component organization, biological regulation, cytosol, cytoskeleton, membrane, and protein binding. KEGG pathway enrichment analysis was mainly involved in cell cycle, oocyte meiosis, progesteronemediated oocyte maturation, FoxO signaling pathway, one carbon pool by folate, and Huntington disease (Figure 6).

\section{Analysis of bub genes in the NOA subgroup}

There was no statistically significant difference in the expression of the 20 hub genes between the NOA-MA and NOA-PreMA subgroups of the GSE108886 dataset (Figure 7).

\section{Validation of the bub genes}

With the exception of PPP2R1B, 19 of the 20 hub genes showed lower expression in the NOA samples of GSE9210 as compared to the OA samples, which was consistent with the GSE145467 and GSE108886 datasets (Figure 8).

\section{Integrated network analysis of miRNA-mRNA interactions}

The 20 hub genes were submitted to the online tool, miRwalk 3.0. Based on the identified miRNA-mRNA pairs, we compared the interaction network containing 51 miRNA-mRNA pairs and visualized them with Cytoscape. Our analysis showed that hsa-miR-3666 and hsa-miR130b-3p downregulated CEP55 and DYNLL2; hsa-miR15b-5p and hsa-miR-6838-5p downregulated CEP55 and PPP2R1B; and hsa-miR-195-5p downregulated PPP2R1B and DYNLL2. The miRNA-gene regulatory network is shown in Figure 9 and Table 4.

\section{Discussion}

Spermatogenesis is a complex process, involving 
Table 2 KEGG pathway analysis of common upregulated and downregulated DEGs

\begin{tabular}{|c|c|c|c|c|c|}
\hline Pathway & ID & Count & Fold Enrichment & $P$ value & Genes \\
\hline Focal adhesion & hsa04510 & 6 & 7.155686546 & $1.08 \mathrm{E}-03$ & $\begin{array}{l}\text { LAMA2, COL6A3, PDGFRA, IGF1, } \\
\text { COL6A1, SHC1 }\end{array}$ \\
\hline $\begin{array}{l}\text { PI3K-Akt signaling } \\
\text { pathway }\end{array}$ & hsa04151 & 6 & 4.272670807 & $9.99 \mathrm{E}-03$ & $\begin{array}{l}\text { LAMA2, COL6A3, PDGFRA, IGF1, } \\
\text { COL6A1, GNG11 }\end{array}$ \\
\hline $\begin{array}{l}\text { ECM-receptor } \\
\text { interaction }\end{array}$ & hsa04512 & 3 & 8.471674877 & $4.52 \mathrm{E}-02$ & LAMA2, COL6A3, COL6A1 \\
\hline $\begin{array}{l}\text { Protein digestion and } \\
\text { absorption }\end{array}$ & hsa04974 & 3 & 8.375405844 & 4.62E-02 & COL6A3, CPA3, COL6A1 \\
\hline $\begin{array}{l}\text { Glycolysis/ } \\
\text { gluconeogenesis }\end{array}$ & hsa00010 & 7 & 5.5713294 & $1.46 \mathrm{E}-03$ & $\begin{array}{l}\text { GAPDHS, LDHC, LDHAL6B, PFKP, } \\
\text { PGAM2, PDHA2, PGK2 }\end{array}$ \\
\hline Cell cycle & hsa04110 & 8 & 3.44036009 & $8.15 \mathrm{E}-03$ & $\begin{array}{l}\text { CCNB2, CDC14A, DBF4, TTK, PTTG1, } \\
\text { CCNA1, CDC25C, MCM4 }\end{array}$ \\
\hline $\begin{array}{l}\text { Progesterone-mediated } \\
\text { oocyte maturation }\end{array}$ & hsa04914 & 6 & 3.677626303 & $2.26 \mathrm{E}-02$ & $\begin{array}{l}\text { PGR, SPDYA, MAPK1, CCNB2, CCNA1, } \\
\text { CDC25C }\end{array}$ \\
\hline $\begin{array}{l}\text { AMPK signaling } \\
\text { pathway }\end{array}$ & hsa04152 & 7 & 3.034789185 & $2.68 \mathrm{E}-02$ & $\begin{array}{l}P P P 2 R 1 B, C P T 1 B, C A B 39 L, P F K P, \\
C C N A 1, P P P 2 R 2 B, P P P 2 R 3 C\end{array}$ \\
\hline $\begin{array}{l}\text { Glucagon signaling } \\
\text { pathway }\end{array}$ & hsa04922 & 6 & 3.231853418 & $3.68 \mathrm{E}-02$ & $\begin{array}{l}\text { LDHC, CPT1B, LDHAL6B, PPP3R2, } \\
\text { PGAM2, PDHA2 }\end{array}$ \\
\hline $\begin{array}{l}\text { Protein processing in } \\
\text { endoplasmic reticulum }\end{array}$ & hsa04141 & 8 & 2.524287877 & $3.78 \mathrm{E}-02$ & $\begin{array}{l}\text { HSPA1L, NGLY1, STT3B, DNAJC5B, } \\
\text { UBQLNL, HSPA4L, UBQLN3, DNAJC5G }\end{array}$ \\
\hline $\begin{array}{l}\text { Biosynthesis of amino } \\
\text { acids }\end{array}$ & hsa01230 & 5 & 3.703165375 & 4.46E-02 & PFKP, PGAM2, TKTL2, PRPS1L1, PGK2 \\
\hline
\end{tabular}

KEGG, Kyoto Encyclopedia of Genes and Genomes; DEGs, differentially expressed genes; Count, number of DEGs.

spermatogonial proliferation (mitosis), spermatocyte meiosis, and spermatid differentiation (14). In this study, we integrated the GSE145467 and GSE108886 datasets and utilized bioinformatics methods to identify 816 common DEGs, including 52 common upregulated and 764 common downregulated genes in NOA. GO enrichment analysis showed that common upregulated DEGs were mainly associated with ECM organization, extracellular exosome, and receptor binding. Common downregulated DEGs were mainly associated with spermatogenesis, nucleus, and protein serine/threonine kinase activity. KEGG pathway analysis showed that common upregulated DEGs were mainly involved in focal adhesion, PI3K-Akt signaling pathway, glioma, ECM-receptor interaction, and protein 


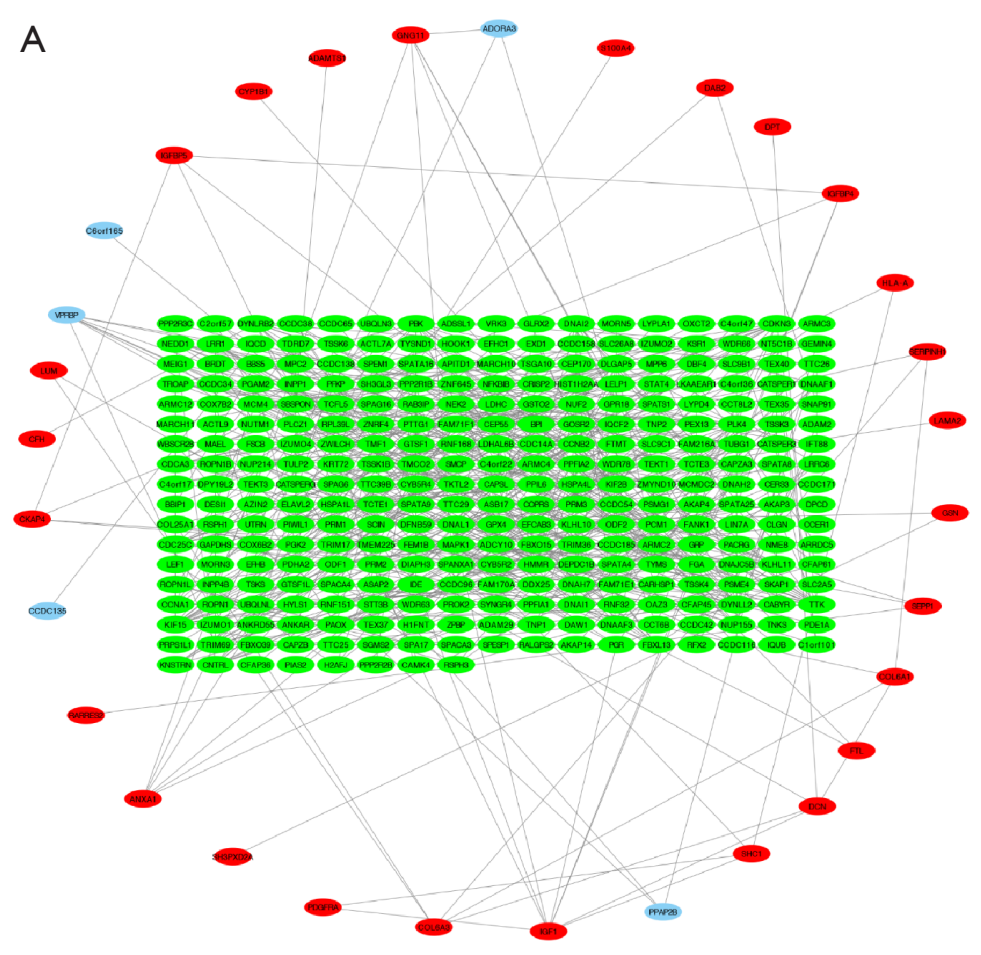

B

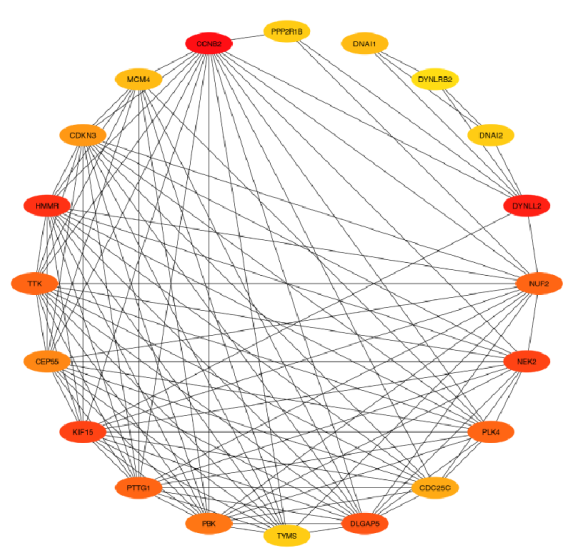

Figure 4 PPI network analysis and hub genes in the protein-protein interaction network. (A) The PPI network for common DEGs. Red circle denotes common upregulated genes; green circle denotes common downregulated genes. (B) The top 20 hub genes in Degree score from the cytoHubba. Redder color indicates higher degree. PPI, protein-protein interaction; DEGs, differentially expressed genes.

digestion and absorption. Common downregulated DEGs were mainly involved in oocyte meiosis, glycolysis/ gluconeogenesis, cell cycle, progesterone-mediated oocyte maturation, AMPK signaling pathway, glucagon signaling pathway, metabolic pathways, protein processing in endoplasmic reticulum, and biosynthesis of amino acids. Most of the above KEGG pathways are involved in spermatogenesis (15-17). However, the two pathway names of "oocyte meiosis" and "progesterone-mediated oocyte maturation" are derived from their roles in female fertility, but genes involved in the "oocyte meiosis" pathway also play an important role in sperm meiosis (18). Similarly, we speculated that genes involved in the "progesteronemediated oocyte maturation" pathway may also play a role in spermatogenesis. These common DEGs may, therefore, be closely related to spermatogenic disorder in NOA patients.

We then identified 20 hub genes: CCNB2, DYNLL2, HMMR, NEK2, KIF15, DLGAP5, NUF2, TTK, PLK4, PTTG1, PBK, CEP55, CDKN3, CDC25C, MCM4, DNAI1, TYMS, PPP2R1B, DNAI2, and DYNLRB2, which were all downregulated genes. Now we summarize the several hub genes that have been studied (Table 5), and then we will describe and discuss them in detail.

CCNB2 is a member of the cyclin B family, which contributes to $G 2 / M$ transition in both mitosis and meiosis (19). Research has found CCNB2 to be continuously expressed in the medaka testis during the process of spermatogenesis (28). CDC25C is a member of the CDC25 family of protein phosphatases, which affects the G2/M phase transition of the cell cycle by activating CDC2 (26). One study reported that under the influence of seleniteinduced oxidative stress, CDC25C was downregulated and p21 (a kinase inhibitor) increased (29). This resulted in the downregulation of the CDC2/cyclin B1 complex that regulates the G2/M phase checkpoint, thereby causing cell cycle arrest in male Balb/c mice (29). Lin et al. (30) examined the testicular messenger RNA (mRNA) transcription levels of 29 patients with NOA, including 18 patients with successful sperm extraction and 11 patients with failed sperm extraction. They found that the mRNA transcription levels of CCNB2 and CDC25C were significantly reduced in NOA patients with failed sperm extraction (30). This further indicates that $\mathrm{CCNB} 2$ and $\mathrm{CDC} 25 \mathrm{C}$ might play 
Table 3 Degree values and descriptions of the top 20 hub genes

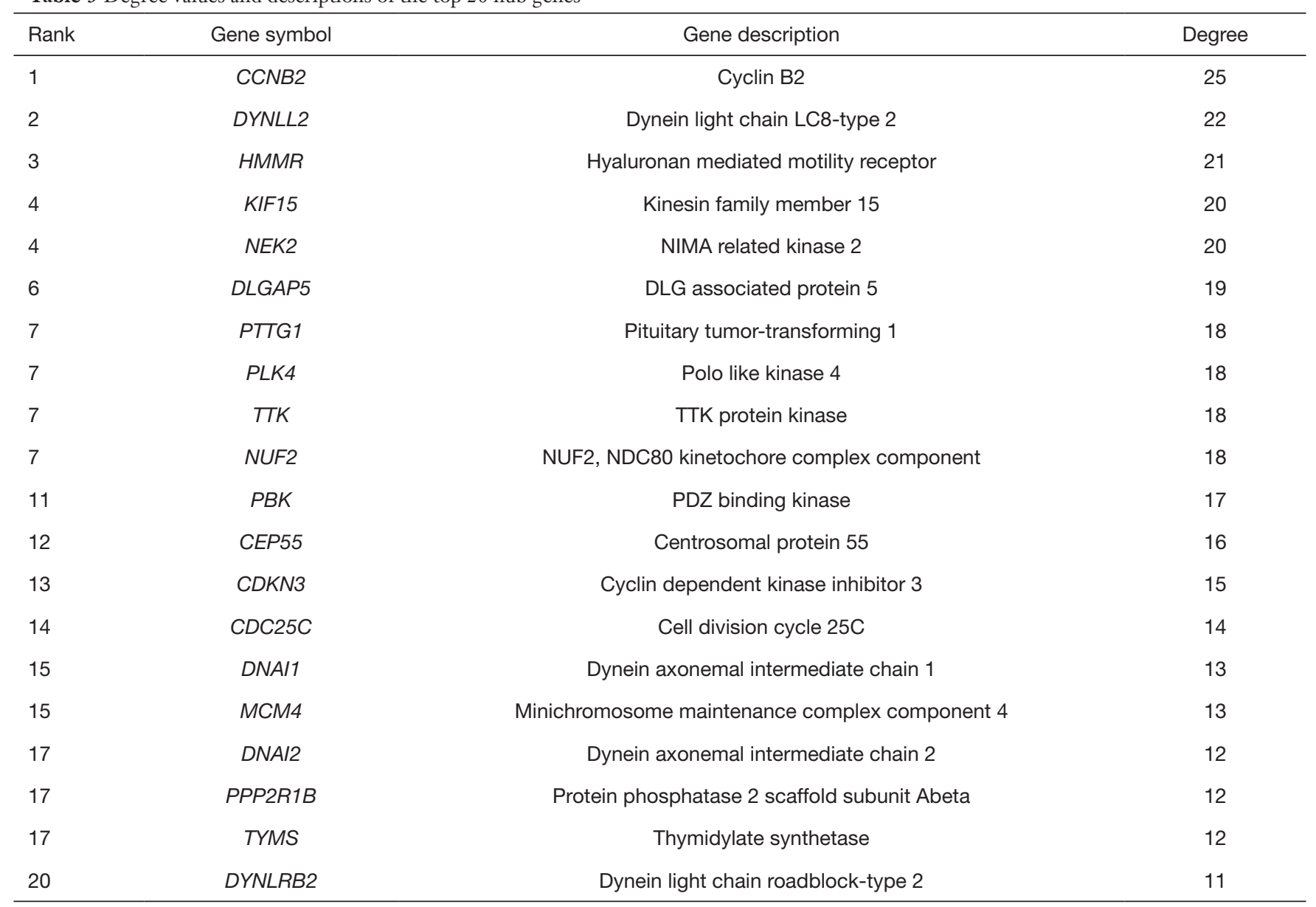
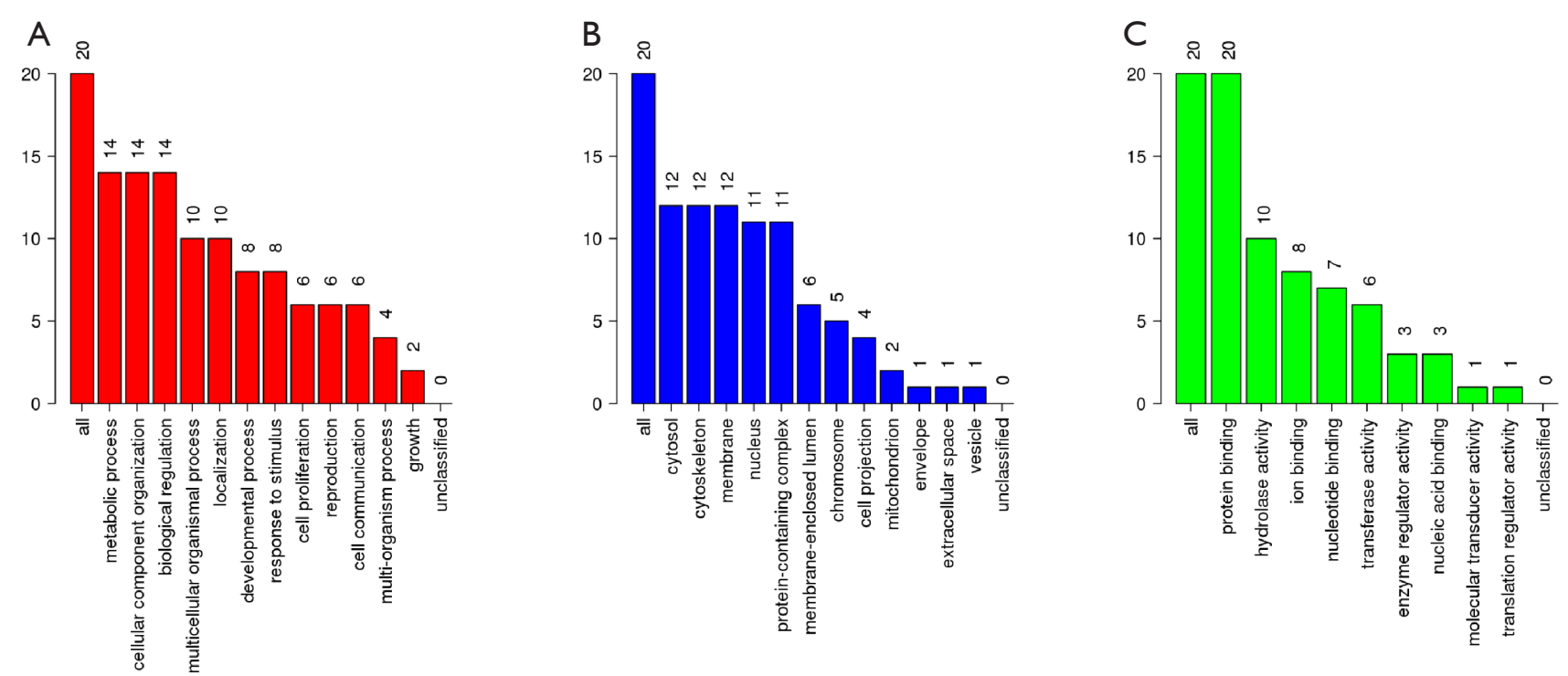

Figure 5 GO map of 20 hub genes. (A) Biological process categories. (B) Cellular component categories. (C) Molecular function categories. GO, Gene ontology. 




Figure 6 KEGG pathway analysis of 20 hub genes. KEGG, Kyoto Encyclopedia of Genes and Genomes.
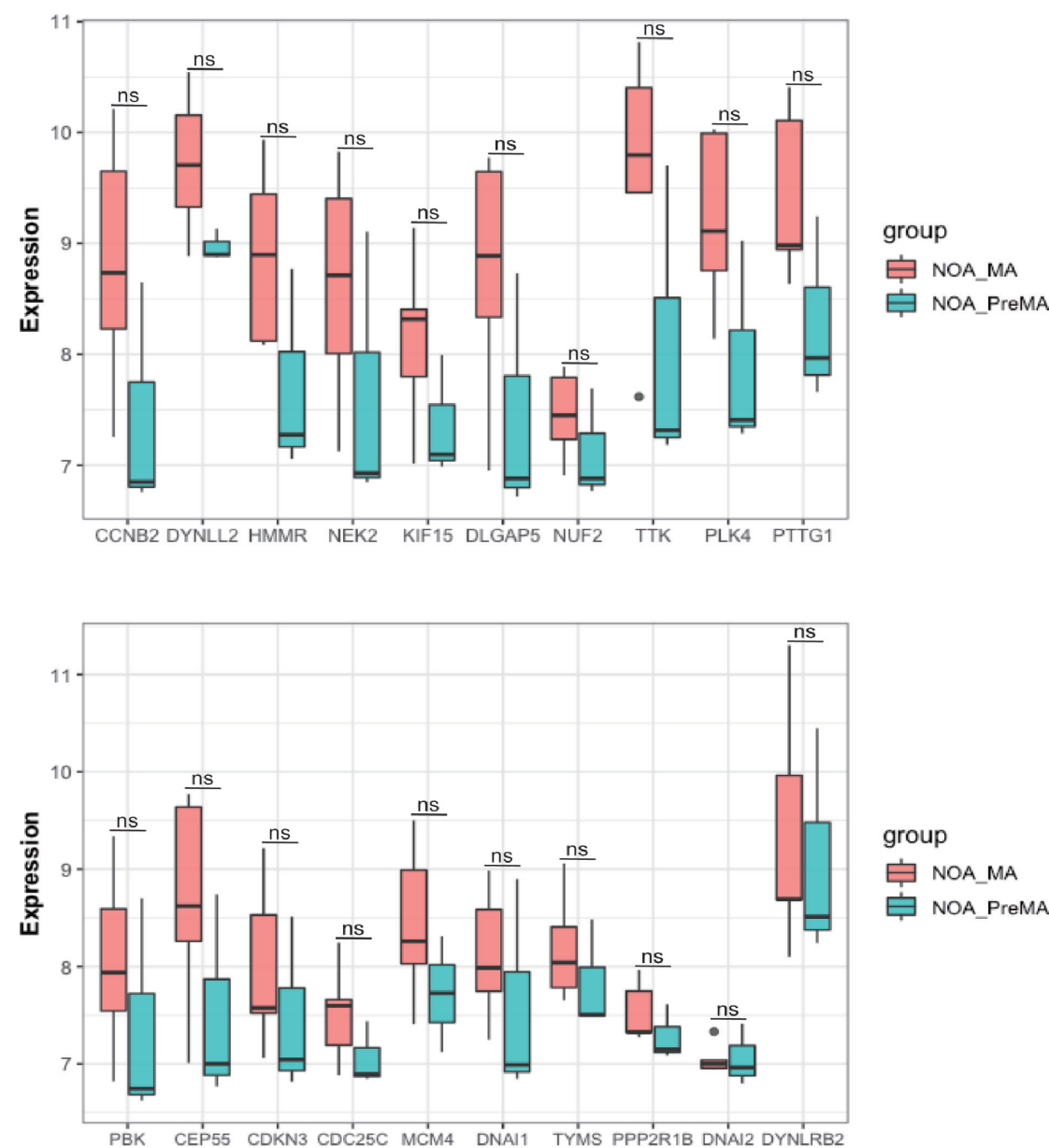

group

官 NOA_MA

由 NOA_PrEMA

Figure 7 The differential expression level of 20 hub genes between NOA-MA and NOA-PreMA subgroups of NOA in GSE108886 dataset (*, $\mathrm{P}<0.05)$. NOA-MA, non-obstructive azoospermia with meiotic arrest; NOA-PreMA, non-obstructive azoospermia with pre-meiotic arrest. 

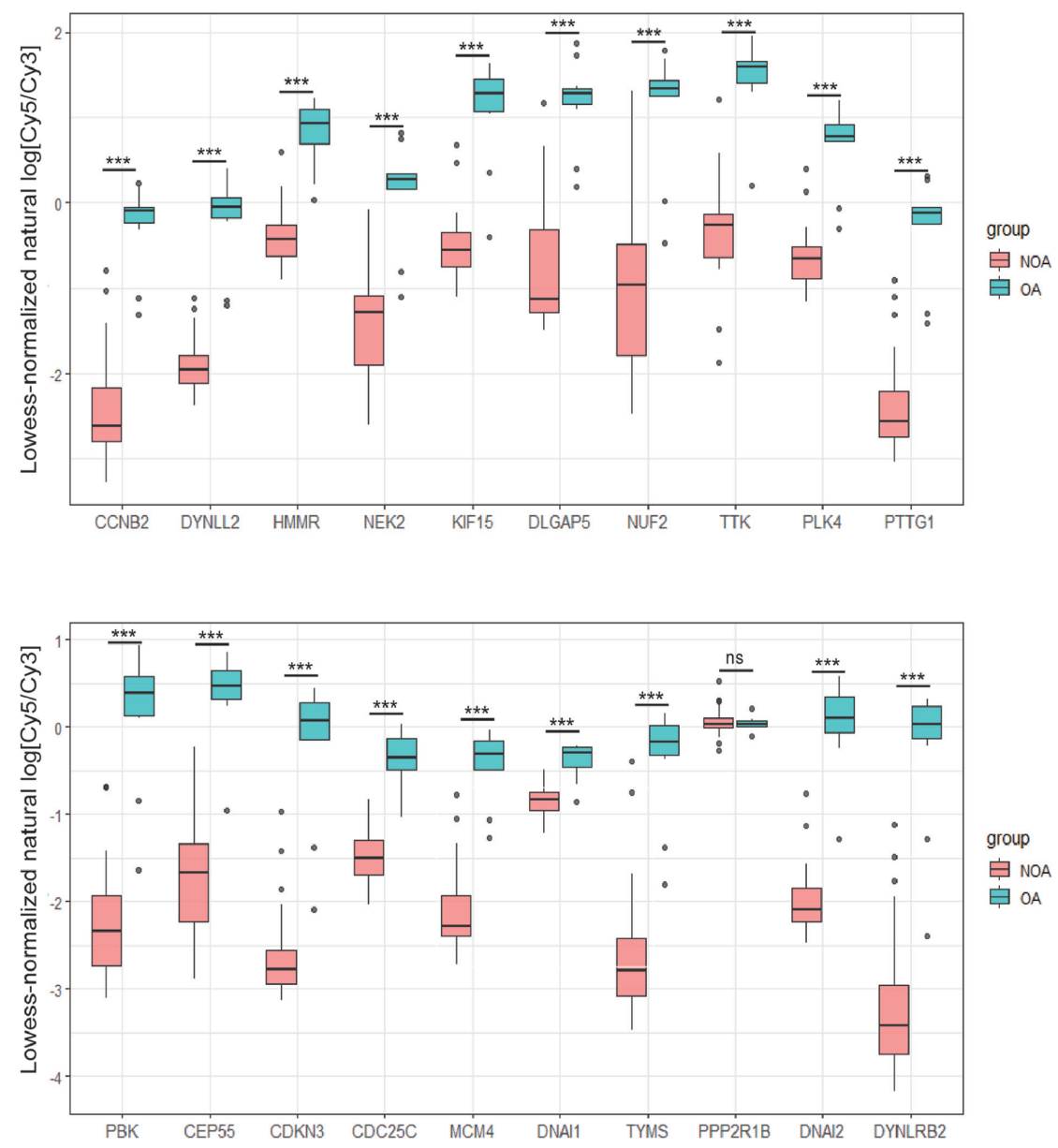

Figure 8 The differential expression level of 20 hub genes between NOA and OA groups in GSE9210 dataset. $\left(^{*}, \mathrm{P}<0.05,{ }^{* *}, \mathrm{P}<0.01,{ }^{* * *}\right.$, $\mathrm{P}<0.001)$. NOA, non-obstructive azoospermia; OA, obstructive azoospermia.

an essential role in spermatogenesis. Hyaluronan-mediated motility receptor (HMMR), also known as receptor for hyaluronan-mediated motility (RHAMM), is a hyaluronic acid-mediated motor receptor (31). One study showed that the downregulation of HMMR is related to a decrease in sperm count, motility, and number of sperm with normal morphology (20). Meanwhile, NIMA-related kinase 2 (Nek2) is a serine/threonine kinase associated with G2/ $M$ phase transition of the cell cycle (21). In the testes of Oreochromis niloticus, Nek2 was generally found to be expressed in primary and secondary spermatocytes (21). Another study reported that Nek2 plays an important role in chromatin condensation during meiosis in male mice (32). TTK (or Mps1) is a dual specificity protein kinase with the ability to phosphorylate tyrosine, serine, and threonine. In reproductive tissues of male zebrafish, $\mathrm{mps}^{\mathrm{zp} 1}$ mutation was found to reduce mitotic checkpoint activity, resulting in abnormal chromosomes in male germ cells, and severe developmental defects in aneuploid progeny (22). Pololike kinases (Plks) are a conserved family of mitotic serine-threonine protein kinases that play a key role in centrosome function (33). PLK4 is a member of the Plks family and necessary for centriole duplication. In a study of PLK4 mutation, the majority of spermatids in Drosophila could not form flagella due to a lack of centrioles, while the depletion of PLK4 in human cells was also seen to induce apoptosis due to mitotic abnormalities (23). PLK4 mutations might also be associated with human Sertoli cell - only syndrome (SCOS) $(34,35)$. PDZ binding kinase $(\mathrm{PBK})$ is a serine/threonine protein kinase, which was found in the outer cell layer of spermatogenic tubules by in situ hybridization, indicating that it plays an important role in the process of spermatogenesis (24). CEP55 (Centrosomal protein 55$)$, located in the centrosomes of interphase 


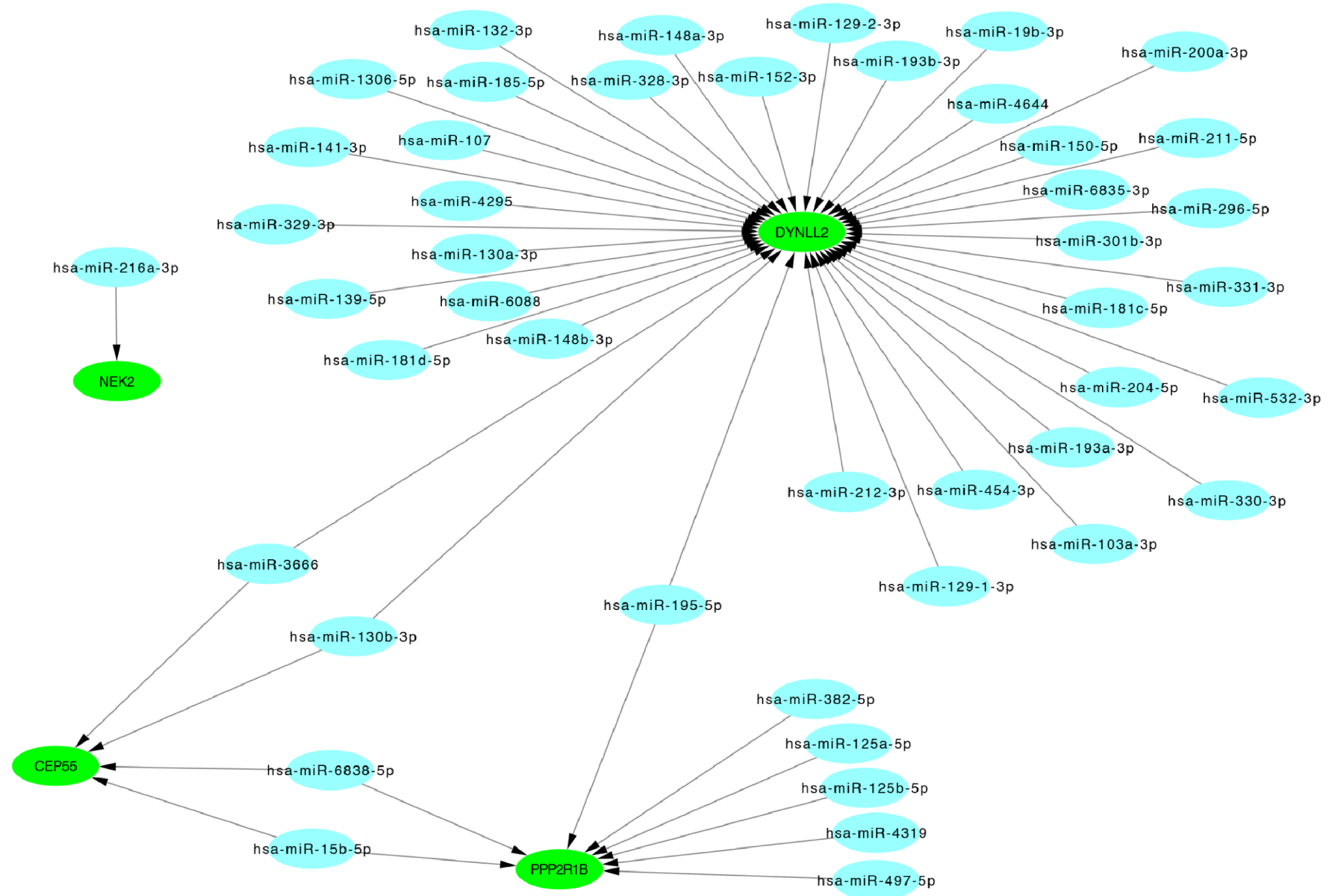

Figure 9 The miRNA-gene regulated network, green color: down- regulated hub genes, blue color: miRNAs.

Table 4 Hub genes and corresponded target miRNAs

\begin{tabular}{ll}
\hline Gene & miRNA \\
\hline NEK2 & hsa-miR-216a-3p \\
CEP55 & hsa-miR-130b-3p hsa-miR-6838-5p hsa-miR-3666 hsa-miR-15b-5p \\
PPP2R1B & hsa-miR-382-5p hsa-miR-497-5p hsa-miR-125a-5p hsa-miR-6838-5p hsa-miR-15b-5p hsa-miR-195-5p hsa-miR-125b- \\
& $5 p$ hsa-miR-4319 \\
DYNLL2 & hsa-miR-193a-3p hsa-miR-148a-3p hsa-miR-139-5p hsa-miR-329-3p hsa-miR-330-3p hsa-miR-296-5p hsa-miR-19b-3p \\
& hsa-miR-129-2-3p hsa-miR-204-5p hsa-miR-4644 hsa-miR-1306-5p hsa-miR-130a-3p hsa-miR-130b-3p hsa-miR-150- \\
& $5 p$ hsa-miR-6088 hsa-miR-181c-5p hsa-miR-185-5p hsa-miR-148b-3p hsa-miR-193b-3p hsa-miR-195-5p hsa-miR-200a- \\
& 3p hsa-miR-211 -5p hsa-miR-454-3p hsa-miR-331-3p hsa-miR-141-3p hsa-miR-103a-3p hsa-miR-129-1-3p hsa-miR- \\
& $212-3 p$ hsa-miR-328-3p hsa-miR-301b-3p hsa-miR-3666 hsa-miR-4295 hsa-miR-532-3p hsa-miR-152-3p hsa-miR-181d- \\
& $5 p$ hsa-miR-107 hsa-miR-132-3p hsa-miR-6835-3p
\end{tabular}

cells, plays an important role in maintaining stable germ cell intercellular bridges during spermatogenesis and spermiogenesis in mice (25). In one study, the expression level of CEP5 5 in patients with maturation arrest was significantly lower than in patients with normal spermatogenesis (36). However, CEP55 overexpression 
Table 5 Several hub genes play a functional role in spermatogenesis

\begin{tabular}{lll}
\hline Gene & Function & Reference \\
\hline CCNB2 & G2/M transition in both mitosis and meiosis & Baker et al. (19) \\
HMMR & Sperm count, motility and number of sperm with normal morphology & Abu-Halima et al. (20) \\
Nek2 & G2/M phase transition of the cell cycle & Matsuoka et al. (21) \\
TTK & Mitotic checkpoint activity & Poss et al. (22) \\
PLK4 & Necessary for centriole duplication & Bettencourt-Dias et al. (23) \\
PBK & In the outer cell layer of spermatogenic tubules & Zhao et al. (24) \\
CEP55 & Maintaining stable germ cell intercellular bridges & Chang et al. (25) \\
CDC25C & G2/M phase transition of the cell cycle & Nilsson et al. (26) \\
DNAl1 & Ciliary function and ultrastructure & Escudier et al. (27)
\end{tabular}

causes change in the proportion of germ cells in mice, and it manifests as a Sertoli-cell-only-tubule phenotype, resulting in mouse infertility (37). Thymidylate synthase (TYMS) converts deoxyuridine monophosphate (dUMP) into deoxythymidine monophosphate (dTMP) using a 5,10-methylenetetrahydrofolate cofactor (38). The TYMS mutation has been associated with failed post-implantation development of the embryo in mice (38). DNAI1 and DNAI2 are members of the dynein intermediate chain family, and their mutations can lead to abnormalities in respiratory ciliary function and ultrastructure, which are important mechanisms of primary ciliary dyskinesia (PCD) (27). DNAI1 is also highly expressed in the testes (39). In male patients with PCD, infertility can be caused by sperm tail dysmotility (40). The DNAI1 mutation in male Drosophila has been associated with infertility caused by motile sperm (41). However, DNAI2 has not been reported in the literature with regard to spermatogenesis. Nine other hub genes, namely DYNLL2, KIF15, DLGAP5, NUF2, PTTG1, CDKN3, MCM4, PPP2R1B, and $D Y N L R B 2$, have also not been reported.

We compared the expression levels of 20 hub genes in the two subgroups (MA and PreMA) of NOA in the GSE108886 dataset and found no statistical difference. This suggests that these hub genes are not affected by NOA classification. This might have been due to the small sample size in the subgroup. A larger number of samples may be needed to confirm this.

In our study, we used WebGestalt for KEGG analysis of 20 hub genes and identified other pathways, including FoxO signaling pathway and Huntington disease. Studies have shown that these pathways are also related to spermatogenesis $(42,43)$.

DYNLL2, CEP55, and PPP2R1B are three important target genes in the miRNA-gene regulatory network. CEP5 5 is described above. DYNLL2 was not involved in any KEGG pathways but could be regulated by most miRNAs, while PPP2R1B was involved in the oocyte meiosis pathway. Among these miRNAs, hsa-miR-3666, hsa-miR-130b-3p, hsa-miR-15b-5p, hsa-miR-6838-5p, and hsa-miR-195-5p have garnered the most research attention. However, these five miRNAs have not been studied in $\mathrm{NOA}$, and further research is required.

A limitation in our study is that the potential miRNAs and hub genes in NOA need to be elucidated through experiments. Further research is required to verify and explore this in-depth.

\section{Conclusions}

In summary, this study involved a comprehensive bioinformatics analysis of DEGs between NOA and OA tissues, and successfully screened 20 hub genes, namely CCNB2, DYNLL2, HMMR, NEK2, KIF15, DLGAP5, NUF2, TTK, PLK4, PTTG1, PBK, CEP5 5, CDKN3, CDC25C, MCM4, DNAI1, TYMS, PPP2R1B, DNAI2, and $D Y N L R B 2$. We also predicted some potential miRNAs of hub genes including hsa-miR-3666, hsa-miR-130b-3p, hsa-miR-15b-5p, hsa-miR-6838-5p, and hsa-miR-195-5p. Some important pathways, including focal adhesion, PI3KAkt signaling pathway, cell cycle, oocyte meiosis, AMPK signaling pathway, FoxO signaling pathway, and Huntington disease, are involved in spermatogenesis. Our results may provide a more detailed understanding of the molecular 
mechanism of NOA and offer potential therapeutic targets for its treatment.

\section{Acknowledgments}

The authors would like to thank the staff members of the GEO database and Yu Du at the Third Affiliated Hospital of Sun Yat-sen University for providing us with technical support.

Funding: This study was funded by the National Natural Science Foundation of China (No. 81571424 \& No. 81771565 ) and the Guangdong Basic and Applied Basic Research Foundation (No. 2019A1515010975).

\section{Footnote}

Reporting Checklist: The authors have completed the STROBE and MDAR reporting checklists. Available at http://dx.doi.org/10.21037/tau-20-1029

Conflicts of Interest: All authors have completed the ICMJE uniform disclosure form (available at http://dx.doi. org/10.21037/tau-20-1029). The authors have no conflicts of interest to declare.

Ethical Statement: The authors are accountable for all aspects of the work in ensuring that questions related to the accuracy or integrity of any part of the work are appropriately investigated and resolved. As all the data in this study were from the GEO public database, the approval of the local ethics committee was not required.

Open Access Statement: This is an Open Access article distributed in accordance with the Creative Commons Attribution-NonCommercial-NoDerivs 4.0 International License (CC BY-NC-ND 4.0), which permits the noncommercial replication and distribution of the article with the strict proviso that no changes or edits are made and the original work is properly cited (including links to both the formal publication through the relevant DOI and the license). See: https://creativecommons.org/licenses/by-nc-nd/4.0/.

\section{References}

1. Devroey P, Fauser BC, Diedrich K, et al. Approaches to improve the diagnosis and management of infertility. Hum Reprod Update 2009;15:391-408.

2. Evers JL. Female subfertility. Lancet 2002;360:151-9.
3. Sharlip ID, Jarow JP, Belker AM, et al. Best practice policies for male infertility. Fertil Steril 2002;77:873-82.

4. Lee JY, Dada R, Sabanegh E, et al. Role of genetics in azoospermia. Urology 2011;77:598-601.

5. Nicopoullos JD, Ramsay JW, Almeida PA, et al. Assisted reproduction in the azoospermic couple. BJOG 2004;111:1190-203.

6. Wu X, Luo C, Hu L, et al. Unraveling epigenomic abnormality in azoospermic human males by WGBS, RNA-Seq, and transcriptome profiling analyses. J Assist Reprod Genet 2020;37:789-802.

7. Maduro MR, Lamb DJ. Understanding new genetics of male infertility. J Urol 2002;168:2197-205.

8. Yao C, Yuan Q, Niu M, et al. Distinct Expression Profiles and Novel Targets of MicroRNAs in Human Spermatogonia, Pachytene Spermatocytes, and Round Spermatids between OA Patients and NOA Patients. Mol Ther Nucleic Acids 2017;9:182-94.

9. Zhang HT, Zhang Z, Hong K, et al. Altered microRNA profiles of testicular biopsies from patients with nonobstructive azoospermia. Asian J Androl 2020;22:100-5.

10. Ferlin A, Raicu F, Gatta V, et al. Male infertility: role of genetic background. Reprod Biomed Online 2007;14:734-45.

11. Hodžić A, Maver A, Plaseska-Karanfilska D, et al. De novo mutations in idiopathic male infertility-A pilot study. Andrology 2020. [Epub ahead of print]. doi: 10.1111/ andr. 12897 .

12. Song E, Song $W$, Ren $M$, et al. Identification of potential crucial genes associated with carcinogenesis of clear cell renal cell carcinoma. J Cell Biochem 2018;119:5163-74.

13. Okada H, Tajima A, Shichiri K, et al. Genome-wide expression of azoospermia testes demonstrates a specific profile and implicates ART3 in genetic susceptibility. PLoS Genet 2008;4:e26.

14. Neto FT, Bach PV, Najari BB, et al. Spermatogenesis in humans and its affecting factors. Semin Cell Dev Biol 2016;59:10-26.

15. Lie PP, Mruk DD, Mok KW, et al. Focal adhesion kinaseTyr407 and -Tyr397 exhibit antagonistic effects on bloodtestis barrier dynamics in the rat. Proc Natl Acad Sci U S A 2012;109:12562-7.

16. Tarnawa ED, Baker MD, Aloisio GM, et al. Gonadal expression of Foxo1, but not Foxo3, is conserved in diverse Mammalian species. Biol Reprod 2013;88:103.

17. Helsel AR, Oatley MJ, Oatley JM. Glycolysis-Optimized Conditions Enhance Maintenance of Regenerative 
Integrity in Mouse Spermatogonial Stem Cells during Long-Term Culture. Stem Cell Reports 2017;8:1430-41.

18. Li B, He X, Zhao Y, et al. Transcriptome profiling of developing testes and spermatogenesis in the Mongolian horse. BMC Genet 2020;21:46.

19. Baker CC, Gim BS, Fuller MT. Cell type-specific translational repression of Cyclin B during meiosis in males. Development 2015;142:3394-402.

20. Abu-Halima M, Ayesh BM, Hart M, et al. Differential expression of miR-23a/b-3p and its target genes in male patients with subfertility. Fertil Steril 2019;112:323-35.e2.

21. Matsuoka Y, Kobayashi T, Kihara K, et al. Molecular cloning of Plk1 and Nek2 and their expression in mature gonads of the teleost fish Nile tilapia (Oreochromis niloticus). Mol Reprod Dev 2008;75:989-1001.

22. Poss KD, Nechiporuk A, Stringer KF, et al. Germ cell aneuploidy in zebrafish with mutations in the mitotic checkpoint gene mps1. Genes Dev 2004;18:1527-32.

23. Bettencourt-Dias M, Rodrigues-Martins A, Carpenter L, et al. SAK/PLK4 is required for centriole duplication and flagella development. Curr Biol 2005;15:2199-207.

24. Zhao S, Dai J, Zhao W, et al. PDZ-binding kinase participates in spermatogenesis. Int J Biochem Cell Biol 2001;33:631-6.

25. Chang YC, Chen YJ, Wu CH, et al. Characterization of centrosomal proteins Cep55 and pericentrin in intercellular bridges of mouse testes. J Cell Biochem 2010;109:1274-85.

26. Nilsson I, Hoffmann I. Cell cycle regulation by the Cdc25 phosphatase family. Prog Cell Cycle Res 2000;4:107-14.

27. Escudier E, Duquesnoy P, Papon JF, et al. Ciliary defects and genetics of primary ciliary dyskinesia. Paediatr Respir Rev 2009;10:51-4.

28. Mita K, Ohbayashi T, Tomita K, et al. Differential Expression of Cyclins B1 and B2 during Medaka (Oryzias latipes) Spermatogenesis. Zoolog Sci 2000;17:365-74.

29. Kaushal N, Bansal MP. Inhibition of CDC2/Cyclin B1 in response to selenium-induced oxidative stress during spermatogenesis: potential role of $\mathrm{Cdc} 25 \mathrm{c}$ and $\mathrm{p} 21$. Mol Cell Biochem 2007;298:139-50.

30. Lin YM, Teng YN, Chung CL, et al. Decreased mRNA transcripts of $M$-phase promoting factor and its regulators in the testes of infertile men. Hum Reprod 2006;21:138-44.

31. Kornovski BS, McCoshen J, Kredentser J, et al. The regulation of sperm motility by a novel hyaluronan receptor. Fertil Steril 1994;61:935-40.

32. Di Agostino S, Fedele M, Chieffi P, et al. Phosphorylation of high-mobility group protein A2 by Nek2 kinase during the first meiotic division in mouse spermatocytes. Mol Biol
Cell 2004;15:1224-32.

33. Barr FA, Sillje HH, Nigg EA. Polo-like kinases and the orchestration of cell division. Nat Rev Mol Cell Biol 2004;5:429-40.

34. Miyamoto T, Bando Y, Koh E, et al. A PLK4 mutation causing azoospermia in a man with Sertoli cell-only syndrome. Andrology 2016;4:75-81.

35. Miyamoto T, Minase G, Shin T, et al. Human male infertility and its genetic causes. Reprod Med Biol 2017;16:81-8

36. Zhu Y, Liu J, Zhang W, et al. CEP55 may be a potential therapeutic target for non-obstructive azoospermia with maturation arrest. Nan Fang Yi Ke Da Xue Xue Bao 2019;39:1059-64.

37. Sinha D, Kalimutho M, Bowles J, et al. Cep55 overexpression causes male-specific sterility in mice by suppressing Foxo1 nuclear retention through sustained activation of PI3K/Akt signaling. FASEB J 2018;32:4984-99.

38. Ching YH, Munroe RJ, Moran JL, et al. High resolution mapping and positional cloning of ENU-induced mutations in the Rw region of mouse chromosome 5. BMC Genet 2010;11:106.

39. Pennarun G, Escudier E, Chapelin C, et al. Lossof-function mutations in a human gene related to Chlamydomonas reinhardtii dynein IC78 result in primary ciliary dyskinesia. Am J Hum Genet 1999;65:1508-19.

40. Storm van's Gravesande K, Omran H. Primary ciliary dyskinesia: clinical presentation, diagnosis and genetics. Ann Med 2005;37:439-49.

41. Fatima R. Drosophila Dynein intermediate chain gene, Dic61B, is required for spermatogenesis. PLoS One 2011;6:e27822.

42. Huang P, Zhou Z, Shi F, et al. Effects of the IGF-1/ PTEN/Akt/FoxO signaling pathway on male reproduction in rats subjected to water immersion and restraint stress. Mol Med Rep 2016;14:5116-24.

43. Hannan AJ, Ransome MI. Deficits in spermatogenesis but not neurogenesis are alleviated by chronic testosterone therapy in R6/1 Huntington's disease mice. J Neuroendocrinol 2012;24:341-56.

Cite this article as: Hu T, Luo S, Xi Y, Tu X, Yang X, Zhang H, Feng J, Wang C, Zhang Y. Integrative bioinformatics approaches for identifying potential biomarkers and pathways involved in non-obstructive azoospermia. Transl Androl Urol 2021;10(1):243-257. doi: 10.21037/tau-20-1029 
Table S1 Common DEGs of the GSE145467 and GSE108886 microarray profile datasets

\begin{tabular}{ll}
\hline Common DEGs & Gene names \\
\hline Upregulated (52) & IGF1 DPT LAMA2 LUM C1S ANXA1 DAB2 SERPINH1 SHC1 GPNMB S100A4 SEPP1 TSKU RARRES2 ADAMTS1 PLTP CRYAB IGDCC4 \\
& CPA3 CFH LAG3 MT1M GNG11 ZFP36L2 FTL SH3PXD2A PRRX1 APCDD1 SLFN11 ALDH1A3 NDRG2 PLPP3 GSN ANGPTL2 HLA-A \\
& HS6ST2 PDGFRA COL6A3 CTSK ABI3BP COL6A1 MGP SFRP4 DCN DLK1 IGFBP4 CAMK2N1 SFRP1 IGFBP5 CYP1B1 CKAP4 ARHGDIB
\end{tabular}

Downregulated SPINK2 USP29 SAXO1 SAMD4A SPESP1 C4orf45 C9orf116 CARHSP1 CCDC116 INSL6 GAPDHS DNAJB8 C17orf97 MYBL1 NEK11 CCNA1 (764) TESMIN PFKP MFSD6L DIAPH3 HERPUD2 TTTY2 INTS7 FAM183A NUP155 ARMC3 REC114 OPLAH LRRC6 AMZ2 PACRG JARID2-AS1 SPACA5 MEX3B ZNF385D ODF2 DEPDC1B H2AFJ TGIF2LY DYRK3 LRRC71 C15orf48 SLC25A2 SLC2A5 IGF2BP3 DNAI1 CCDC110 IQCK COPRS TCP11 C4orf22 CCDC74B KDM4D CLGN FLJ35934 CALR3 ADGB IZUMO4 C17orf74 PGK2 CLHC1 NOL4 C16orf92 SPRR2C SKAP1 SESN3 DEPDC7 CABS1 CFAP36 RAB3IP SOCS7 NUP214 CMTM2 LYAR CCT8L2 ZSCAN5A NUP210L DNAH7 LINC00905 PIAS2 KRT72 TERB2 HIST1H2AA KIF2B MNS1 NUPR2 PRR30 ANKMY1 ARHGAP9 PCYT2 ACOT13 C9orf43 PPP1R2P9 FAM209A GK2 ARL4A EXD1 CCDC34 ST7L ADAM29 INPP4B CDCA3 LINC00919 MARCH10 TCP10L SPATA25 DPP10-AS3 CNBD2 YBX2 CDC14A UTRN ACTL7B ACSBG2 PPEF1 RNFT1 MAPK1 LYZL1 PRPS1L1 RMDN2 IQCF2 H1FNT KIAA1683 ATPIF1 ODF1 SPAG16 C10orf62 C3orf30 MEIG1 HMGB4 TSKS ZMYND15 RNF32 LYPLA1 PROK2 RPGRIP1 TEKT1 C20orf85 HSPA1L LYPD4 DNAJC5G C10rf158 CT83 C10rf101 TTLL6 PCSK4 CFAP126 KLHDC3 CFAP206 DNAAF3 PPFIA2 PPP3R2 IZUMO1 RFPL3S SPATA17 IQCF1 CFAP61 SLC26A8 NUTM1 LDHAL6B BBOF1 CMTM1 CCNY LINC00608 FAM187B BOLL C11orf88 CFAP157 DFNB59 PIH1D2 TEX35 DRICH1 TMIGD3 TIAM2 CEP170 C17orf64 EFHB GLRX2 CCNB2 ZNF451 AKAP14 TRIM17 TPGS2 TBPL1 TEX40 UBQLN3 FGA ACTRT2 ULK4 PXDNL CNTRL CCDC65 LAMP3 DESI1 BANF2 LYZL4 ROPN1B FAM182B CFAP53 LRIT1 RFX3 GMCL1 SOHLH2 SCIN WDR87 FAM163A RNF148 ZDHHC20 PSMG1 CEP55 LEMD1 PDZD8 C11orf97 TNP2 FEM1B C12orf50 SLC36A3 SPZ1 ABHD2 TMEM99 TMCO5A TTC39B MAEL PHYHIPL SEC14L1 FAM71E1 MLF1 NME8 LRRC37B ZPBP2 AKAP3 C40rf17 CCDC185 FSCN3 ANKAR SLC12A6 CAPN11 SPACA4 C9orf50 ADAM32 MPP6 FTMT LINC01096 NGLY1 TMCO2 PAOX SLC9C1 DNAJC5B MBD3L1 KHDRBS3 SCP2D1 CCDC38 MRVI1-AS1 C7orf62 C2orf57 KLK5 PLCZ1 PRSS58 EFHC1 KNSTRN PCM1 STAT4 DNAJB3 ZFAND4 FAM216A HCFC2 ACTL7A COIL BRDT TUBG1 TMEM217 SWT1 CDC25C FANCD2OS TTC21A TMEM38B C20orf144 C4orf47 FAM47B MROH7 COX7B2 FAM24A CCDC83 PIWIL1 LINC00221 LRRCC1 ACTL9 CCDC136 TSGA10 C1orf111 HINT3 IZUMO2 CATSPERG SPACA3 FSCB UBQLNL HABP4 RGS22 RALGPS2 C9orf131 LDHC RNF113B C6orf10 PDHA2 SPAM1 SGMS2 HYLS1 PPP2R1B CAPZA3 SPANXA1 SLC22A16 TTC29 TEX37 FAM71E2 ZWILCH MCMDC2 OXCT2 LRWD1 ZBBX MCM4 BIRC8 SPEM1 IFT88 CCDC169 LARP1B SPA17 LYL1 C12orf10 SLC25A19 CAMK4 SPATA31C1 PROM1 HSPA4L KIF15 PDZD9 SPATA4 ALDH1A2 SHCBP1L GTSF1L HDAC11 DBF4 CXorf51B MTTP RNF133 CATSPER3 SLFNL1 DNAAF1 TCTE3 FAM221B TEKT3 CDR2 BPIFA3 C10rf105 EFCAB6 C10orf107 CCDC96 ADTRP SETD9 NFKBIB LCA5L PGR FAR2 CCDC173 GRK4 COX6B2 PRPH SPATA19 DLGAP5 TCTE1 C4orf36 FNDC11 CFAP161 CTAGE15 OAZ3 BBS5 PHTF1 TKTL2 RSPH10B IRGC LINC00301 SPATS1 CYB5R2 BSCL2 ASAP2 CCDC138 SAMD15 TSSK4 FAM71F1 GOSR2 AZIN2 CAPSL UBTD2 GGN ZMYND12 BTBD16 LEF1 IQUB ATP23 C9orf24 DYNLL2 CCDC15 CABYR KSR1 C11orf63 TTC25 C2orf70 GTSF1 CETN1 TDRD10 C6orf201 CST13P AKIRIN1 ICA1 CTAGE6 PTTG3P CATSPER1 ST6GALNAC2 DYDC1 OSBP2 OSCP1 GSTO2 KRTDAP LRRFIP2 OR7E156P ANKRD13A DEUP1 CARNMT1 SYPL1 KLHL10 TSSK1B TTC23L MYCBPAP C1Orf194 SEPT12 MYH3 RSPH1 ZNRF4 RAB11FIP5 TEX33 ROCK1P1 SH3GL3 TSACC ABCC12 PPFIA1 ELAVL2 HMMR MRPS15 PPP2R2B WISP3 CCDC54 TTC12 MPC2 ARRDC5 C10rf100 WBSCR28 LRRC56 APH1B CFAP52 CCDC158 CFAP43 TMEM191B ACRBP CYB5R4 DRC7 ZCCHC13 GCNT3 C5orf46 FAM170A PPP2R3C SBSPON CAB39L PEX13 HSFY2 MORN3 ATP5S HEMGN GPR135 GRP C12orf56 ROPN1 C6orf99 CST8 CASC1 KYNU CPT1B LIN7A MS4A5 DCAF1 CCDC171 FAM205A STAMBPL1 LRR1 HOOK1 FABP12 TTC39C SPATA6 TSSK3 LRRC46 SPATA22 BEND7 PPM1G ZSCAN29 CDNF OR10J1 SPACA9 SPAG6 ERICH6 ZC2HC1C INPP1 PHOSPHO2 ARMC4 TSPAN16 CCDC42 RND2 TP53TG3 C17orf80 OLAH MROH2B C7orf34 CCDC187 FAM220A IQCF5 VRK3 SWI5 SMCP ZDHHC19 TCFL5 FAM71A SUN3 PRM2 LRRC34 TTLL2 TDRD7 NDUFAF7 RNF151 C2orf42 NSUN7 PRSS37 TNKS CXorf65 ATP8B3 CLDND2 C12orf60 ITPRIPL1 ZNF689 PMS1 AGBL5 LINC00264 KLHL11 FAM181A EQTN STK33 TEX36-AS1 CPXCR1 WDR78 DDX25 C20orf141 AXDND1 PARD6A GKAP1 GPX4 TSGA13 SPATA9 TSSK6 SYNGR4 SPANXD FAM186B DAW1 LYZL2 NRAV TYMS SFT2D2 HSF5 DNAL1 TEX29 RNF168 TRIM69 TBC1D26 USP2 CSNK2A2 HRASLS5 C1orf94 IDE FAM209B TRIM42 DPY19L2 ALS2CR12 IQCD CCT6B DKKL1 OVOL2 SSUH2 ASB17 GEMIN4 ROPN1L CCDC89 GUCA1A SLC9B1 NT5C1B DNAJC4 ASRGL1 TMPRSS12 PRM3 BBIP1 C10orf82 C11orf65 KLK12 TNP1 TRIM59 C2orf88 C1orf87 LYZL6 SPATA32 LKAAEAR1 SERP2 TSNAXIP1 PMFBP1 PGAM2 TYSND1 DEF6 C8orf88 ERICH3 CCIN ZNF233 ARMC12 HYPM TMEM225 LINC00635 MORC1 TUBB3 RIOK3 PPM1E ADSSL1 SLC6A16 TTLL10 TEX26 COL25A1 MAST4 BPI NEDD1 CCER1 ANKRD55 MARCH11 SPERT LELP1 TMEM31 TIMD4 CCDC70 FBXO39 SPDYA APITD1 CRISP2 CERS3 HGFAC CCDC7 PLK4 PRM1 LINC00347 GPR18 MALL C22orf23 KLHDC9 BTG4 TTK FANK1 RSPH3 DRG1 PFN4 LRRC73 SIRPD SPACA7 CDKN3 CCDC178 SPATA8 MORN5 ZNF683 DYNLRB2 DNAI2 NUF2 PBK FAM92A1 CCDC112 PZP ZBTB32 C2orf16 USP44 ATL3 KPNA5 AKAP4 SNAP91 TMF1 EFCAB3 ZNF829 TRIM36 MAP3K19 FBXL13 C9orf135 ALS2CR11 ADAM2 SPATA16 ALKBH7 C11orf70 FAM104A HRASLS GLIPR1L1 ADCY10 FAM217A SPATA7 HIPK1 MDH1B TTC16 SI LRRC3B ZC3H14 PDE1A PTTG1 TPPP2 UBE2DNL NEK2 ASGR1 SIGLECL1 TPD52L3 MS4A6E CAPZB TROAP PSME4 IL7 ZMYND10 LRRC36 CCDC189 WDR66 FBXO15 MORC2-AS1 ZPBP MS4A14 PP2D1 EFCAB11 PROCA1 LINC00854 ZNF645 GALNTL5 XKR3 PPIL6 TTC26 STT3B SLCO6A1 ADAD1 DNAH2 TMEM89 SRSF12 DPCD RFX2 CFAP45 ARMC2 TULP2 LINC01548 TBATA ABHD1 RPL39L SPATA24 WDR63 ZNF165 TP53TG5 FAM71C C12orf54

Common DEGs, common differentially expressed genes. 